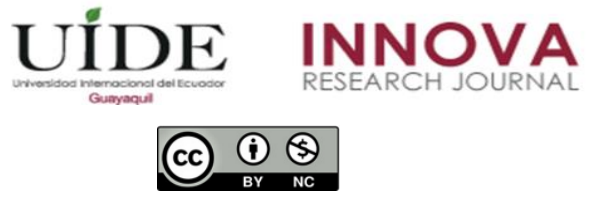

INNOVA Research Journal, ISSN 2477-9024

(Enero - Abril 2021). Vol. 6, No.1 pp. 18-30

DOI: https://doi.org/10.33890/innova.v6.n1.2021.1490

URL: http://revistas.uide.edu.ec/index.php/innova/index

Correo: innova@uide.edu.ec

\title{
Análisis de la gestión administrativa y la calidad del servicio en la atención de los partos humanizados en un hospital nacional de Lima
}

\section{Analysis of administrative management and service quality in the care of humanized births in a national hospital in Lima}

Susana Aliaga Díaz

(D) https://orcid.org/0000-0002-4495-0994

Universidad César Vallejo, Perú

Noel Alcas Zapata

(iD https://orcid.org/0000-0001-9308-4319

Universidad César Vallejo, Perú

Autor para correspondencia: susana.aliaga_diaz@hotmail.com; noelz75@hotmail.com

Fecha de recepción: 05 de agosto de 2020 - Fecha de aceptación: 09 de septiembre de 2020

\section{Resumen}

Este trabajo tuvo como objetivo encontrar la influencia de la gestión administrativa y la calidad del servicio de un hospital público de la ciudad de Lima en los partos humanizados, estudio cuantitativo, descriptivo, causal y transversal. Se trabajó con una muestra de 90 pacientes de una población de 117 mujeres que se atendieron en dos meses seguidos; muestreo aleatorio. Se utilizaron tres instrumentos validados por expertos y cuya confiabilidad se midió con Alfa de Cronbach. En los resultados se evidenció que existe incidencia de la gestión administrativa y la calidad de servicio en el parto humanizado.

Palabras claves: gestión administrativa, calidad de servicio, parto humanizado.

\begin{abstract}
The objective of this work was to find the influence of the administrative management and the quality of the service of a public hospital in the city of Lima in the humanized deliveries, quantitative, descriptive, quasal and transversal study. We worked with a sample of 90 patients from a population of 117 women who were treated in two consecutive months; random sampling. Three instruments validated by experts and whose reliability was measured with Cronbach's Alpha were used. The results showed that there is an incidence of administrative management and quality of service in humanized delivery.
\end{abstract}

Keywords: administrative management, quality of service, humanized delivery. 


\section{Introducción}

Desde hace algunos años la gestión administrativa es medida debido a la relevancia que ha tomado, los resultados que se obtienen en estas mediciones permite entender las razones por la que unas empresas son capaces de cumplir con sus objetivos y otras no. En la administración pública es igual, los gobiernos necesitan conocer sus resultados para plantear acciones de mejora que los lleve a una mayor eficiencia, este es el caso de Perú. De acuerdo con esto, la gestión administrativa podría ayudar a entender los problemas que se asocian a la salud de los peruanos en general, este estudió tuvo como objetivo principal entender la incidencia entre la gestión administrativa, la calidad en los servicios y el parto humanizado que se ve afectado por distintos factores como las políticas, aspectos sociales, problemas económicos y culturales que influyen los hospitales nacionales y sus desempeños. En este orden de ideas, se buscó analizar la gestión administrativa, calidad de servicio y su posible influencia en los procesos de partos humanizados, de hospital público, analizando el caso del Hospital Nacional Carlos Lanfranco La Hoz, que se ubica en el distrito de Puente Piedra.

En el plano nacional, existe la necesidad de contar con instituciones hospitalarias eficientes y transparentes. En ese sentido el (Acuerdo Nacional, 2016) impulsó la implementación de la gestión pública por objetivos, eficiente y con transparencia, y sobre todo con rendición de cuentas al control ciudadano. El Plan Nacional Concertado de Salud, tiene como meta fundamental reducir el porcentaje de muertes en las madres gestantes en un $75 \%$, lo que no se concreta aún con los esfuerzos que hace el Estado peruano (Ministerio de Salud, 2007).

Este trabajo tuvo como objetivo entender la realidad del Hospital Nacional Carlos Lanfranco La Hoz y proponer soluciones que puedan mejorar la gestión administrativa, la calidad del servicio y los procesos inherentes al parto humanizado; permitiendo una reflexión relevante para los académicos y profesionales del área de estudio.

En esta investigación se han revisado antecedentes fuera de Perú, uno de los primeros propuso una reflexión sobre la naturaleza y la evidencia científica que se generan en torno al parto y la lactancia materna, apoya la idea que la humanización de la parto y destaca la lactancia materna (Alzuguir y Nucci, 2015). Por otra parte, otros estudios concluyeron que socialmente el parto es visto como un escenario de peligro y es importante el trato que reciben de los profesionales que las asisten en estos casos (Biurrun, 2017; Silverstri, 2016). En un trabajo argentino, encontraron un incremento considerable en el desarrollo institucional gracias a la gestión de políticas públicas (Cardozo y Bulcourf, 2016), otro estudio afirmó que los problemas financieros y administrativos de un hospital público generalmente se deben a la falta de ética y al deficiente manejo de la información (Balcazar, 2019).

Cueva y Rodríguez, 2017, encontraron en su estudio que hay una responsabilidad compartida entre la empresa privada y el gobierno por las políticas multisectoriales, lo que lleva a la reflexión sobre los empresarios y los gobernantes como gestores de vida. En Brasil en la investigación realizada por Araujo, Tressa, Santos, Olivera y Pinto, 2019 demostraron que las mujeres que optan por los partos humanizados lo hacen con la convicción que este es la mejor opción para garantizar la vida de su hijo y la propia. 
En Perú, Parrales, 2016 precisó la falta de evidencias para afirmar la existencia de una posible relación entre las actitudes de los profesionales y el conocimiento que poseen sobre el parto humanizado, Ñahuis, 2017 encontró que la comunicación y el servicio que se les otorga a los familiares de las pacientes son relevante. Otros estudios afirmaron que los usuarios muestran percepciones altas sobre el servicio cuando sienten empatía y seguridad por parte de los médicos y personal que los atienden, tanto en los hospitales como en las consultas privadas (Infantes, 2017; Minaya, 2018; Oré, 2017; Ruiz, 2017; Siadén, 2016).

La teoría humanista de la administración es otra forma de analizar la administración, desde esta perspectiva, el análisis se orienta a las relaciones humanas, analiza el clima laboral y las relaciones grupales, de los trabajadores. La teoría racionalista de la administración incorpora los argumentos de la clásica y de la humanista, poniendo interés en la tecnología para la toma de decisiones, propugna la organización formalizada de los procesos que realizan los trabajadores y el control de sus conductas; no se considera situaciones como las asociaciones de poder, problemas de intereses de grupo y la posible incidencia dentro y fuera de la organización (Agüero, 2007). El fin de la administración es lograr un desempeño organizacional eficiente (Marcó, Loguzzo, y Fedi, 2016). La gestión administrativa es entendida como un proceso en el cual el funcionamiento de la empresa u organización, tiene que aprovechar el buen uso de sus recursos que se dispone, para el logro de los objetivos establecidos (Cano, 2017). García, 2011 definió que la administración actualmente se fundamenta en la teoría y enseñanza con referentes diversos: desde un enfoque racionalista (instrumental) y otro positivista, acentuando el funcionalismo de carácter utilitario que en la práctica constituye una base epistemológica del pensamiento y el saber de la administración que se le suele llamar tradicional.

La calidad de servicio, tiene el enfoque de la gestión de la calidad que es utilizada para referirse a un sistema que puede relacionar un conjunto de variables que se consideran relevantes para la aplicación de los elementos y métodos que posibiliten la mejora en la calidad. En ese sentido se distinguen tres dimensiones muy marcadas: los principios que orientan las acciones organizativas, las practicas que consisten en la concreción de actividades y las técnicas que pueden hacer que las practicas sean efectivas (Camison, Cruz, y Gonzalez, 2007). Está orientada a la percepción del usuario (Borré y Vega, 2014) se afirma que es un fenómeno multifactorial subjetivo, (Parasuraman, Berry, y Zeithaml, 1991) se ha definido a calidad de servicio como la divergencia entre expectativas sobre la atención recibida. Por lo que la calidad del servicio permanecerá en el nivel alto, cuando las diferencias negativas entre lo esperado y lo percibido sean minimizadas. Llinás (2010) mencionado por (Borré y Vega, 2014) sostuvo que la calidad del servicio se entiende como las tipologías que reconocen a las insuficiencias del usuario y la ausencia de deficiencias(Camisón, Cruz, y González, 2006). La satisfacción es entendida como el resultado de la evaluación que el cliente hace del servicio recibido, y que está en estrecha relación de manera con el servicio esperado (Mejías y Manrique, 2011).

Por otra parte, la calidad implica contrastación de los resultados y el estándar establecido en las organizaciones. Es decir, se trata de comparar el resultado parcial o total, durante un proceso frente a los requerimientos llamados estándares, como lo sostuvieron (Sanabria, Romero, y Flórez, 2015). También Campanella (1998), citado por (Manrique, Manrique, Chávez, y Manrique, 2018) afirmó que la calidad se concreta no solo con dar un concepto, sino 
como filosofía de evaluación de todos los servicios que se brindan o en los producto que se venden, estableciendo una comparación con productos similares.

Sobre los procesos o partes de la calidad de servicio, se tiene la propuesta de (Parasuraman et al., 1991) quienes en 1988, propusieron dimensiones que permiten medirla, posteriormente gracias a otra investigación en cinco empresas de USA establecieron el constructo con cinco dimensiones para medir dicha variable.

Para (Parasuraman et al., 1991) los elementos tangibles, son aquellos que están asociados al servicio brindado, como los equipos, infraestructura instalada y recursos humanos. La fiabilidad, es reconocida como una destreza para hacer la prestación que se ha propuesto de manera fiable y prolija (Parasuraman et al., 1991). (Gondres, Báez, Lajes, y Del Castillo, 2013) afirmaron que la confiabilidad es el área de la estadística que se ocupa del análisis de las fallas o errores que aparecen en los componentes a lo largo del tiempo, este concepto se aplica en la actualidad en las ciencias sociales, en las industrias, ciencias médicas, entre otros. La capacidad de respuesta consiste en la actitud o disposición que demuestran los trabajadores de la organización al brindarles el servicio en forma rápida y eficiente. La seguridad, implica que se tengan las personas que atienden a los usuarios deben tener los conocimientos necesarios para generar confianza y credibilidad (Parasuraman et al., 1991).

Se entiende que la empatía, es un proceso mediante el cual se puede atender en forma personalizada a los clientes, es aquí donde se establece una relación afectiva de buen trato y cordialidad, con los usuarios que recurren a solicitar el servicio, tal como lo sostuvieron (Parasuraman et al., 1991).

Las características del parto humanizado, están delimitadas por tres factores: las fuerzas del parto, que es el conjunto de todas las contracciones que se producen en el cuello uterino de la parturienta; el segundo factor es el objeto del parto, que lo constituye el feto y sus anexos que son lo que se debe llevar hasta el exterior, el segundo factor es el conducto del parto, que es el camino que debe recorrer el feto desde el interior de útero hasta el exterior de la madre (Hospital Sanitas La Zarzuela, 2012).

Se entiende que el parto humanizado es una experiencia positiva, que implica un desenlace relevante para todas las mujeres en el proceso o trabajo de parto. Al respecto, la (OMS, 2018), afirmó que el parto humanizado, se entiende como la experiencia que debe superar las afirmaciones y perspectivas de carácter personal y socioculturales anteriores que trae consigo la mujer. El parto humanizado, a diferencia del parto normal, en el cual no se recurre a ningún tipo de medicamento para que la mujer pueda dar a luz, se caracteriza por brindarle a la mujer la asistencia que se llama "parto no intervenido" en el cual se establece una labor de vigilancia, tanto de la mujer como del feto, brindando además asistencia de carácter psicólogo a la parturienta y a su familia por parte de los profesionales del centro médico (Hospital Sanitas La Zarzuela, 2012).

Sobre las dimensiones del parto humanizado, la (OMS, 2018), dentro de sus recomendaciones para la concreción del parto humanizado, sostuvo que se deben considerar los siguientes procesos: (a) atención durante todo el trabajo de parto y el nacimiento, (b) etapa de 
dilatación, (c) etapa expulsivo, (d) alumbramiento, (e) atención al recién nacido, (f) atención de la mujer tras el nacimiento.

\section{Metodología}

Estudio cuantitativo, utiliza a la estadística como su apoyo para el recojo y procesamiento de datos, estudio transversal de lógica deductiva.(Hernández, Fernández, y Baptista, 2014). Descriptivo, explicativo y causal, se buscó explicar la variabilidad de la atención del parto humanizado con respectos a la gestión administrativa y la calidad de servicio (Hernández et al., 2014). Estudio no experimental, porque no existió manipulación alguna de los datos (Anguera et al., 2010). El procedimiento que se utilizó es el método hipotético - deductivo, que se aplica de lo general a lo particular y viceversa (Bernal, 2010).

Este estudio contó con dos variables independientes: Gestión administrativa y Calidad de servicio que ya fueron explicadas teóricamente. La gestión administrativa, en forma operacional se define mediante cinco dimensiones: planificación, organización, dirección, y control. Para lo cual se aplicó un instrumento con 20 ítems. A nivel operacional la calidad de servicio se mide con 05 subvariables o dimensiones; se utilizó un instrumento con opciones de respuesta ordinal o politómicas, con 22 ítems.

Una sola variable dependiente: atención del parto humanizado en forma operacional el parto humanizado se concretó mediante la utilización de un cuestionario con 24 ítems y 03 opciones de respuesta.

La población fue de 117 pacientes que son atendidas en promedio la sala de partos del Hospital Carlos Lanfranco La Hoz mediante dos meses consecutivos. El muestreo fue probabilístico y aleatorio simple (Bernal, 2010). La técnica empleada para el levantamiento de información fue la encuesta, los instrumentos utilizados fueron cuestionarios que fueron validados por expertos.

En la tabla 1 encontramos los resultados obtenidos en la aplicación del Alfa de Cronbach, el cual está reservado para instrumentos que han sido elaborados con escala ordinal politómica tipo Likert. También se consideró, una muestra piloto a 35 pacientes, que no participaron en la muestra de este estudio. Se presentan los resultados del análisis de confiabilidad de las variables en estudio. Se evidencia demás, que el estadístico Alfa de Cronbach para la gestión administrativa es igual a 0,737, en la Calidad del servicio equivale a 0,934 y en la atención del parto humanizado es igual a 0,889 . Por lo tanto, se puede afirmar que los cuestionarios que miden las tres variables, son confiables. 


\section{Tabla 1}

Resultados del análisis de confiabilidad de las variables

\begin{tabular}{lcc}
\hline Variables & Alfa de Cronbach & N $^{\circ}$ de ítems \\
& & 17 \\
\hline Gestión administrativa & 0,737 & 22 \\
Calidad de servicio & 0,934 & 33 \\
Atención del parto humanizado & 0,889 & \\
\hline
\end{tabular}

Para el recojo de los datos inicialmente se utilizó una hoja de Excel, luego fueron exportados al programa SPSS, en el cual, se procesaron y generaron los resultados a nivel descriptivo con tablas y figuras. También, en el nivel inferencial se aplicó la regresión logística multivariable, con las tres variables de estudio. Dado que el objetivo general pretende medir el grado de incidencia que tienen en el parto humanizado las dos primeras variables. Toda esta estrategia, para arribar a las conclusiones se ha logrado teniendo como soporte el método hipotético deductivo, para el proceso de prueba de hipótesis. partiendo de lo general a lo particular como lo sostuvo (Bernal, 2010).

\section{Resultados}

\section{Resultados descriptivos}

\section{Descripción de la gestión administrativa y dimensiones}

\section{Tabla 2}

Niveles y frecuencias de la gestión administrativa

\begin{tabular}{lcc}
\hline Niveles & Frecuencias & Porcentajes \\
\hline Deficiente & 19 & 21,1 \\
Regular & 42 & 46,7 \\
Bueno & 29 & 32,2 \\
Total & 90 & 100,0
\end{tabular}

Observación: Resultados procesados con el SPSS_V25(2020)

La tabla 2 se muestra las frecuencias y niveles de la gestión administrativa, donde el $46,7 \%$ de las usuarias encuestadas perciben dicha variable en el nivel regular, seguido del 32,2\% en el nivel bueno. 


\section{Tabla 3}

Dimensiones de la gestión administrativa

\begin{tabular}{lcccccccc}
\hline \multirow{2}{*}{ Niveles } & \multicolumn{2}{c}{ Planificación } & \multicolumn{2}{c}{ Organización } & \multicolumn{2}{c}{ Dirección } & \multicolumn{2}{c}{ Control } \\
\cline { 2 - 9 } & $\mathrm{F}$ & $\%$ & $\mathrm{~F}$ & $\%$ & $\mathrm{~F}$ & $\%$ & $\mathrm{~F}$ & $\%$ \\
\hline Deficiente & 22 & 24,4 & 32 & 35,6 & 42 & 46,7 & 30 & 33,3 \\
Regular & 40 & 44,4 & 13 & 14,4 & 37 & 41,1 & 21 & 23,3 \\
Bueno & 28 & 31,1 & 45 & 50,0 & 11 & 12,2 & 39 & 43,3 \\
Total & 90 & 100,0 & 90 & 100,0 & 90 & 100,0 & 90 & 100,0 \\
\hline
\end{tabular}

Observación: Resultados procesados con el SPSS_V25(2020)

En la tabla 3 y se observa los niveles y frecuencias de las dimensiones. La dimensión planeación se presenta como regular con el 44,4\% de las percepciones de las usuarias, la organización es buena y representa el 50,0\%. Asimismo, la dimensión dirección está en el nivel deficiente con el 46,7\%, seguido del $41,1 \%$ que es regular. También se tiene el control, que ubica como bueno, con el 43,3\% de las percepciones, seguido del 33,3. del nivel deficiente.

\section{Descripción del parto humanizado y dimensiones}

\section{Tabla 4}

Niveles y frecuencias del parto humanizado

\begin{tabular}{lrc}
\hline Niveles & Frecuencias & Porcentajes \\
\hline Insatisfecho & 30 & 33,3 \\
Aceptable & 35 & 38,9 \\
Satisfecho & 25 & 27,8 \\
Total & 90 & 100,0 \\
\hline
\end{tabular}

Observación: Resultados procesados con el SPSS_V25(2020)

La tabla 4 se observa las frecuencias y niveles de la atención del parto humanizado, donde el 38,9\% de las usuarias encuestadas perciben dicha variable en el nivel aceptable, seguido del 33,3\% en el nivel insatisfecho.

\section{Tabla 5}

Dimensiones del parto humanizado

\begin{tabular}{|c|c|c|c|c|c|c|c|c|c|c|c|c|}
\hline \multirow[t]{2}{*}{ Niveles } & \multicolumn{2}{|c|}{$\begin{array}{l}\text { Atención del } \\
\text { trabajo de } \\
\text { parto y } \\
\text { nacimiento }\end{array}$} & \multicolumn{2}{|c|}{$\begin{array}{l}\text { Período de } \\
\text { dilatación }\end{array}$} & \multicolumn{2}{|c|}{$\begin{array}{r}\text { Período } \\
\text { expulsivo }\end{array}$} & \multicolumn{2}{|c|}{ Alumbramiento } & \multicolumn{2}{|c|}{$\begin{array}{l}\text { Atención al } \\
\text { recién nacido }\end{array}$} & \multicolumn{2}{|c|}{$\begin{array}{l}\text { Atención de la } \\
\text { mujer tras el } \\
\text { nacimiento }\end{array}$} \\
\hline & $\mathrm{F}$ & $\%$ & $\mathrm{~F}$ & $\%$ & $\mathrm{~F}$ & $\%$ & $\mathrm{~F}$ & $\%$ & $\mathrm{~F}$ & $\%$ & $F$ & $\%$ \\
\hline Insatisfecho & 40 & 44,4 & 40 & 44,4 & 33 & 36,7 & 38 & 42,2 & 25 & 27,8 & 34 & 37,8 \\
\hline Aceptable & 31 & 34,4 & 39 & 43,3 & 28 & 31,1 & 37 & 41,1 & 40 & 44,4 & 40 & 44,4 \\
\hline Satisfecho & 19 & 21,1 & 11 & 12,2 & 29 & 32,2 & 15 & 16,7 & 25 & 27,8 & 16 & 17,8 \\
\hline Total & 90 & 100,0 & 90 & 100,0 & 90 & 100,0 & 90 & 100,0 & 90 & 100,0 & 90 & 100,0 \\
\hline
\end{tabular}

Observación: Resultados procesados con el SPSS_V25(2020) 
En la tabla 5 se observa los niveles y frecuencias de las partes o aspectos de la atención del parto humanizado. La dimensión atención del trabajo de parto y nacimiento está en el nivel insatisfecho con el 44,4\% de las percepciones de las usuarias, la dimensión período de dilatación se ubica en el nivel insatisfecho y representa el 44,4\%. Asimismo, la dimensión periodo expulsivo se ubica en el nivel insatisfecho con el 36,7\%, seguido del 32,2\% en el nivel satisfecho. También se tiene a la dimensión alumbramiento, que ubica en el nivel insatisfecho, con el 42,2\% de las percepciones, seguido del 41,1\%. del nivel aceptable. Por otra parte, la dimensión atención al recién nacido representa el 44,4\% y es aceptable, Finalmente, la atención de la mujer tras el parto y nacimiento está en el nivel aceptable con el 44,4\%.

\section{Descripción de la calidad de servicio y dimensiones}

\section{Tabla 6}

Niveles y frecuencias de la calidad de servicio

\begin{tabular}{lcc}
\hline Niveles & Frecuencias & Porcentajes \\
\hline Insatisfecho & 28 & 31,1 \\
Aceptable & 41 & 45,6 \\
Satisfecho & 21 & 23,3 \\
Total & 90 & 100,0 \\
\hline
\end{tabular}

Observación: Resultados procesados con el SPSS_V25(2020)

La tabla 6 muestra las frecuencias y niveles de la calidad de servicio, donde el 45,6\% de las usuarias encuestadas perciben dicha variable en el nivel aceptable, seguido del $31,3 \%$ en el nivel insatisfecho.

\section{Tabla 7}

\section{Dimensiones de la calidad del servicio}

\begin{tabular}{|c|c|c|c|c|c|c|c|c|c|c|}
\hline \multirow[t]{2}{*}{ Niveles } & \multicolumn{2}{|c|}{$\begin{array}{l}\text { Elementos } \\
\text { tangibles }\end{array}$} & \multicolumn{2}{|c|}{ Fiabilidad } & \multicolumn{2}{|c|}{$\begin{array}{l}\text { Capacidad de } \\
\text { respuesta }\end{array}$} & \multicolumn{2}{|c|}{ Seguridad } & \multicolumn{2}{|c|}{ Empatía } \\
\hline & $\mathrm{F}$ & $\%$ & $\mathrm{~F}$ & $\%$ & $\mathrm{~F}$ & $\%$ & $\mathrm{~F}$ & $\%$ & $\mathrm{~F}$ & $\%$ \\
\hline Insatisfecho & 25 & 27,8 & 40 & 44,4 & 41 & 45,6 & 32 & 35,6 & 20 & 22,2 \\
\hline Aceptable & 38 & 42,2 & 37 & 41,1 & 24 & 26,7 & 28 & 31,1 & 30 & 33,3 \\
\hline Satisfecho & 27 & 30,0 & 13 & 14,4 & 25 & 27,8 & 30 & 33,3 & 40 & 44,4 \\
\hline Total & 90 & 100 & 90 & 100,0 & 90 & 100,0 & 90 & 100,0 & 90 & 100,0 \\
\hline
\end{tabular}

Nota: Análisis estadístico SPSS_V25(2020)

En la tabla 7 se observan los niveles y frecuencias de las dimensiones de la calidad de servicio. La dimensión elementos tangibles está en el nivel aceptable con el 42,2\% de las percepciones de las usuarias, la dimensión fiabilidad se ubica en el nivel insatisfecho y representa el 44,4\%. Asimismo, la dimensión capacidad de respuesta se ubica en el nivel insatisfecho con el 45,6\%, seguido del27,82\% en el nivel satisfecho. También se tiene a la dimensión seguridad, que ubica en el nivel insatisfecho, con el 35,6\% de las percepciones, 
seguido del 33,3\% del nivel aceptable. Por otra parte, la dimensión empatía representa el 44,4\% y está en el nivel satisfecho.

Este estudio a partir de la revisión del estado del arte generó una hipótesis general que fue analizada con la regresión lineal multivariado, en la general se definió:

$\mathrm{H}_{0}$ : $\quad$ No existe incidencia de la gestión administrativa y calidad de servicio en el parto humanizado de un hospital nacional de Lima.

$\mathrm{H}_{1}$ : $\quad$ Existe incidencia de la gestión administrativa y calidad de servicio en el parto humanizado de un hospital nacional de Lima.

\section{Tabla 8}

Contraste de la razón de verosimilitud

\begin{tabular}{lcccc}
\hline & Criterios de ajuste de modelo & \multicolumn{2}{c}{ Pruebas de la razón de verosimilitud } \\
\cline { 2 - 5 } & $\begin{array}{c}\text { Logaritmo de la verosimilitud -2 de } \\
\text { Efecto }\end{array}$ & & & \\
\hline modelo reducido & Chi-cuadrado & gl & Sig. \\
Intersección & $31,107^{\mathrm{a}}$ &, 000 & 0 &. \\
Gestión administrativa & 36,572 & 45,464 & 4 &, 000 \\
Calidad de servicio & 71,124 & 40,017 & 4 &, 000 \\
\hline
\end{tabular}

Nota: Análisis estadístico SPSS_V25(2020)

En la tabla 8, se observa los resultados de las pruebas de verosimilitud, entre la gestión administrativa y la calidad del servicio en la atención del parto humanizado. Se evidencia que la significancia para las variables independientes es igual a ,000 <, 001, lo cual permite concluir que los modelos del parámetro son iguales a cero, según, $\mathrm{H}_{0}$.

La tabla 9, muestra la calidad de ajuste del modelo, mediante la prueba Pseudo $\mathrm{R}$ cuadrado con los coeficientes de variabilidad Cox y Snell, Nagelkerke y McFadden. Se observa además que, en ningún caso, se aproximan los valores a 1; sin embargo, son mayores a 0,2. Lo cual permite afirmar que el ajuste es bueno. En ese sentido, el coeficiente de McFadden está en el rango $0 \leq \mathrm{R} 2 \leq 1$, pero evidentemente, no se aproxima a 1, dado que se considera que el ajuste es bueno cuando está en el rango de $0,2 \leq \mathrm{R} 2 \leq 0,4$, por lo que se tiene una buena calidad de ajuste porque su valor es igual a 0,257. Por estas razones, es conveniente usar para explicar la calidad del modelo al coeficiente de Nagelkerke que es igual a 0,484 , lo cual prueba que este modelo presenta un ajuste bueno. Es decir que, este modelo es predictor de los niveles del variable efecto, demostrado con el coeficiente de Nagelkerke en la prueba Pseudo R cuadrado, indica que el 48,4\% de la variabilidad de la atención del parto humanizado, depende de la gestión administrativa y de la calidad de servicio. 


\section{Tabla 9}

Calidad de ajuste del modelo

\begin{tabular}{rrr}
\hline Cox y Snell & Nagelkerke & McFadden \\
\hline, 429 &, 484 &, 257
\end{tabular}

Nota: Análisis estadístico SPSS_V25(2020)

\section{Tabla 10}

Información de ajuste de modelo

\begin{tabular}{lrrrr} 
& \multicolumn{2}{c}{ Criterios de ajuste de modelo } & \multicolumn{3}{c}{ Pruebas de la razón de verosimilitud } \\
Modelo & Logaritmo de la verosimilitud -2 & Chi-cuadrado & gl & Sig. \\
\hline Sólo intersección & 81,504 & & & \\
Final & 31,107 & 50,397 & 8 &, 000 \\
\hline
\end{tabular}

Nota: Análisis estadístico SPSS_V25(2020)

En la tabla 10, se muestra la bondad de ajuste en donde el estadístico de prueba Chicuadrado de ajuste es igual a 50,397 y la significancia $\mathrm{p}=0,000<0,01$, lo cual indica, además, que el modelo en su totalidad a la regresión logística multivariante en forma significativa. Esto, evidencia un modelo con predictores. Por consiguiente, se puede afirmar que estos resultados explican la presencia de la significancia como resultado de la contrastación de las variables explicativas, considerando la significancia teórica de 0,05 y nivel de confianza de 95\%, lo cual indica que, el modelo es este caso, presenta un adecuado ajuste de calidad. Consiguientemente, se rechaza $\mathrm{H}_{0}$ y se acepta el supuesto que existe incidencia de la gestión administrativa y calidad de servicio en el parto humanizado de un hospital nacional de Lima.

\section{Conclusión y Recomendación}

En síntesis, con respecto al objetivo general se concluye que, existe incidencia de la gestión administrativa y calidad de servicio en el parto humanizado de un hospital nacional de Lima. Esto se verifica con la prueba contraste de verosimilitud que indicó como valor de significancia 0,000 menor a 0,05. Asimismo, el coeficiente de Nagelkerke indica que el 48,4\% de la variabilidad del parto humanizado, depende de la gestión administrativa y calidad de servicio. Se recomienda que las autoridades del hospital, remodelar las instalaciones físicas, equipamiento de forma moderna y atractiva de acuerdo a las necesidades establecidas para parto digno y saludable de madre y niño. Respetando los derechos de la mujer a vivir un proceso de parto que respeten sus deseos y necesidades, sus tiempos biológicos y psicológicos sin intervenciones masivas. Este remodelado de las instalaciones implica mejor infraestructura instalada y mejores equipos a utilizar en el proceso de parto humanizado. 
Se sugiere a otros investigadores realizar estudios similares en hospitales privados para poder establecer comparativos y evidenciar si la tipología podría afectar los resultados, de la misma forma establecer muestras que incluyan hospitales similares más de uno a la vez para obtener resultados que pudieran ser comparados entre sí.

\section{Referencias Bibliográficas}

Acuerdo Nacional. (2016). Políticas De Estado Y Planes De Gobierno 2016- 2021 (1ra.). Lima. Retrieved from http://acuerdonacional.pe/wp-content/uploads/2016/03/Politicas-deEstado-y-Planes-de-Gobierno-2016_2021.pdf

Agüero, J. O. (2007). Teoría de la administración: Un campo fragmentado y multifacético. Revista Científica "Visión de Futuro," 7(1). Retrieved from https://www.redalyc.org/pdf/3579/357935466001.pdf

Alzuguir, F. V., \& Nucci, M. F. (2015). Maternidade mamífera? Concepções sobre natureza e ciência em uma rede social de mães. Mediações - Revista de Ciências Sociais, 20(1), 217. https://doi.org/10.5433/2176-6665.2015v20n1p217

Anguera, M. T., Arnau, J., Ato, M., Martínez, R., Pascual, J., \& Vallejo, G. (2010). Métodos de investigación en psicología. Madrid: Síntesis. Retrieved from https://www.casadellibro.com/libro-metodos-de-investigacion-en-psicologianddsc/9788477382843/468956

Araújo, B. R. O. de, Trezza, M. C. S. F., Santos, R. M. dos, Oliveira, L. L. F. de, \& Pinto, L. M. T. R. (2019). Entre ritos y contextos: Resoluciones y significados atribuidos al parto natural humanizado. Cultura de Los Cuidados Revista de Enfermería y Humanidades, 23(54), 206-216. https://doi.org/10.14198/cuid.2019.54.18

Balcazar, A. M. (2019). Gobernanza corporativa, una propuesta para el mejoramiento en la gestión administrativa y financiera en el hospital E.S.E Nuestra Señora del Carmen Tabio Cundinamarca. Contaduría Universidad de Antioquia, 73, 13-32. https://doi.org/10.17533/udea.rc.n73a01

Bernal, C. (2010). Metodologia de la Investigación. (Pearson Educación, Ed.) (3ra.). Colombia. Retrieved from http://biblioteca.uccvirtual.edu.ni

Biurrun, A. (2017). La humanización de la asistencia al parto: Valoración de la satisfacción, autonomía y del autocontrol. (Tesis doctoral). Universitat de Barcelona. Retrieved from https://www.tesisenred.net/bitstream/handle/10803/457137/ABG_TESIS.pdf?sequence=1 \&isAllowed $=\mathrm{y}$

Borré, Y. M., \& Vega, Y. (2014). Calidad percibida de la atención de enfermería por pacientes hospitalizados, 23(3), 81-94. Retrieved from https://scielo.conicyt.cl/pdf/cienf/v20n3/art_08.pdf

Camison, C., Cruz, S., \& Gonzalez, T. (2007). Gestión de la calidad: conceptos, enfoques, modelos y sistemas. https://doi.org/8420542628

Cano, C. A. (2017). La administración y el proceso administrativo. Universidad de Bogotá. Retrieved from http://www.eumed.net/libros/img/portadas/1665.pdf

Cardozo, N. D., \& Bulcourf, P. (2016). El desarrollo institucional de la Administración y Políticas Públicas en Argentina. Un estudio de la enseñanza desde el proceso democratizador hasta nuestros días. Estudios Políticos (Medellín),

(49). 
https://doi.org/10.17533/udea.espo.n49a12

Cuevas, R., \& Rodríguez, R. (2017). Responsabilidad social y ética profesional en la gestión de la administración pública y empresarial. Pensamiento \& Gestión, (42), 26. https://doi.org/10.14482/pege.41.9704

García, O. H. (2011). Una aproximación a la gerencia del siglo XXI. Cuadernos de Administracion, 27(45), 153-172. https://doi.org/10.25100/cdea.v27i45.450

Gondres, I., Báez, R., Lajes, S., \& Del Castillo, A. (2013). Determinación de la confiabilidad en interruptores de potencia: Caso de estudio. Ingeniare, 21(2), 271-278. https://doi.org/10.4067/S0718-33052013000200010

Hernández, R., Fernández, C., \& Baptista, P. (2014). Metodología de la investigación. McGRAWHILL / INTERAMERICANA EDITORES, S.A. DE C.V. (6 $\left.6^{\mathrm{a}}\right)$. México, D.F.

Hospital Sanitas La Zarzuela. Conoce todo lo importante sobre el parto humanizado, Sanitas 24 (2012). Retrieved from https://www.hospitallazarzuela.es/pdf/parto-humanizado.pdf

Hospital SAnitas la Zarzuela. (2012). Parto Humanizado: Conoce todo lo importante sobre el parto humanizado. Sanitas, 24. Retrieved from https://www.hospitallazarzuela.es/pdf/partohumanizado.pdf

Infantes, F. M. (2017). Calidad de atención y grado de satisfacción de los usuarios de consulta externa del Centro de Atención de Medicina Complementaria del Hospital III Iquitos-2016. Revista Peruana De Medicina Integrativa, 2(2), 133-139. https://doi.org/10.1016/j.ceca.2003.11.008

Manrique, J., Manrique, C., Chávez, B., \& Manrique, J. E. (2018). Calidad técnica y percibida del servicio de operatoria dental en una clínica dental docente. Rev Estomatol Herediana, 28(3), 185-194. Retrieved from https://www.researchgate.net/publication/328128753_Calidad_tecnica_y_percibida_del_s ervicio_de_operatoria_dental_en_una_clinica_dental_docente

Marcó, F., Loguzzo, H. A., \& Fedi, J. L. (2016). IntroduccIón a la GestIón y admInIstracIón en las orGanIzacIones. (Universidad Nacional Arturo Jauretche, Ed.) (2a . ed.). Buenos Aires. Retrieved from http://biblioteca.unaj.edu.ar/wpcontent/uploads/sites/8/2017/02/Introduccion-gestion-y-administracionorganizaciones.pdf

Mejías, A. A., \& Manrique, S. (2011). Dimensiones de la satisfaccion de clientes bancarios universitarios: Una aproximación mendiante el analisis de factores. Ingenería Industrial, 32(1), 43-47. Retrieved from http://www.redalyc.org/articulo.oa?id=360433575007\%0ACómo

Minaya, E. (2018). Calidad de Atención y satisfacción del usuario del servicio de consultorio médico de Medicina, según percepción de los pacientes del Hospital de Huaral, 2016. Universidad César Vallejo. Retrieved from http://repositorio.ucv.edu.pe/bitstream/handle/UCV/13225/Minaya_TDOEL.pdf?sequenc $\mathrm{e}=1$ \&isAllowed $=\mathrm{y}$

Ministerio de Salud. (2007). Plan Nacional Concertado de Salud. Retrieved from http://bvs.minsa.gob.pe/local/MINSA/000_PNCS.pdf

Ñahuis, G. (2017). Percepción de la gestante sobre la atención del parto humanizado en SANNA clínica san Borja-enero a marzo, 2017. Universidad César Vallejo. Retrieved from http://repositorio.ucv.edu.pe/bitstream/handle/UCV/24148/Nahuis_AG.pdf?sequence=1\& is Allowed $=\mathrm{y}$

Oré, A. (2017). Gestión y desempeño en un Hospital de Essalud, Cañete, Lima, 2017. Repositorio

Esta obra se comparte bajo la licencia Creative Common Atribución-No Comercial 4.0 International (CC BY-NC 4.0) Revista de la Universidad Internacional del Ecuador. URL: https://www.uide.edu.ec/ 
de la Universidad del Pacífico - UP. Retrieved from http://repositorio.up.edu.pe/bitstream/handle/11354/1968/Andres_Tesis_maestr\%EDa_20 17.pdf;jsessionid=9A7576E258CE51369D44B4ED9CEF71C6? sequence $=1$

Organización Mundial de la Salud (OMS). (2018). Recomendaciones de la OMS Para los cuidados durante el parto , Transformar la atención a mujeres y neonatos para. Departamento de Salud Reproductiva e Investigaciones Conexas, Organización Mundial de La Salud, WHORHR-18(8), 1-8. Retrieved from https://www.who.int/reproductivehealth/publications/intrapartum-care-guidelines/es/

Parasuraman, A., Berry, L. L., \& Zeithaml, V. A. (1991). Refinement and reassessment of the SERVQUAL scale. Article in Journal of Retailing, 67(4), 420-450. Retrieved from https://www.researchgate.net/publication/304344168

Parrales, E. (2016). Nivel de conocimiento sobre el parto humanizado y su relación con las actitudes de los internos de Obstetricia. Instituto Nacional Materno Perinatal. Universidad Nacional Mayor de San Marcos. Retrieved from http://cybertesis.unmsm.edu.pe/bitstream/cybertesis/4981/1/Parrales_me.pdf

Ruiz, R. (2017). Calidad de atención al cliente y el nivel de satisfacción del usuario externo en el Hospital ESSALUD II, Tarapoto, San Martin-2016. Universidad César Vallejo. Retrieved from http://repositorio.ucv.edu.pe/bitstream/handle/UCV/1597/ruiz_pr.pdf?sequence=1

Sanabria, P. E., Romero, V. del C., \& Flórez, C. I. (2015). El concepto de calidad en las organizaciones: una aproximación desde la complejidad. Universidad \& Empresa, 16(27), 165-213. https://doi.org/10.12804/rev.univ.empresa.27.2014.06

Siadén, Q. I. (2016). Calidad de atención en la Consulta Externa del Hospital de San Juan de Lurigancho 2016.2 Retrieved from http://www.repositorioacademico.usmp.edu.pe/bitstream/usmp/2613/1/SIADEN_QI.pdf

Silvestri, N. (2016). Análisis de la percepción de las puérperas sobre la atención del parto humanizado en el Hospital Materno Infantil Comodoro Meisner durante el período de noviembre - diciembre de 2016. Retrieved from https://rephip.unr.edu.ar/bitstream/handle/2133/10191/CEI - MGSSS - Tesis Silvestri Silvia Noemí.pdf?sequence $=3 \&$ isAllowed $=\mathrm{y}$ 